Original Research

\title{
Tannin-Mn coordination polymer coated carbon quantum dots nanocomposite for fluorescence and magnetic resonance bimodal imaging
}

\author{
Weibing Xu $\mathbb{I}^{1} \cdot$ Jia Zhang ${ }^{1} \cdot$ Zhijie Yang $^{2} \cdot$ Minzhi Zhao ${ }^{1} \cdot$ Haitao Long ${ }^{1} \cdot$ Qingfeng $\mathrm{Wu}^{3} \cdot$ Fang Nian ${ }^{1}$
}

Received: 5 July 2021 / Accepted: 16 November 2021 / Published online: 24 January 2022

(c) The Author(s) 2022

\section{Abstract}

The MR/FI bimodal imaging has attracted widely studied due to combining the advantages of MRI and FI can bridge gaps in sensitivity and depth between these two modalities. Herein, a novel MR/FI bimodal imaging probe is facile fabricated by coating the Mn-phenolic coordination polymer on the surface of the carbon quantum dots. The structure of the as-prepared nanocomposite probe is carefully validated via SEM, TEM, and XPS. The content of $\mathrm{Mn}^{2+}$ is calculated through the EDS and TGA. The quantum yield (QY) and emission wavelength of the probe are about $7.24 \%$ and $490 \mathrm{~nm}$, respectively. The longitudinal r1 value $\left(2.43 \mathrm{mM}^{-1} \mathrm{~s}^{-1}\right)$ with low $\mathrm{r} 2 / \mathrm{r} 1$ (4.45) of the probe is obtained. Subsequently, fluorescence and MR imaging are performed. The metabolic pathways in vivo are inferred by studying the bio-distribution of the probe in major organs. Thus, these results indicate that probe would be an excellent dual-modal imaging probe for enhanced MR imaging and fluorescence imaging.

\section{Graphical Abstract}

MR/FI bimodal imaging probe is built via in-situ coated Mn-phenolic coordination polymer on the surface of the carbon quantum dots. The in vitro and vivo image property of the probe is evaluated.

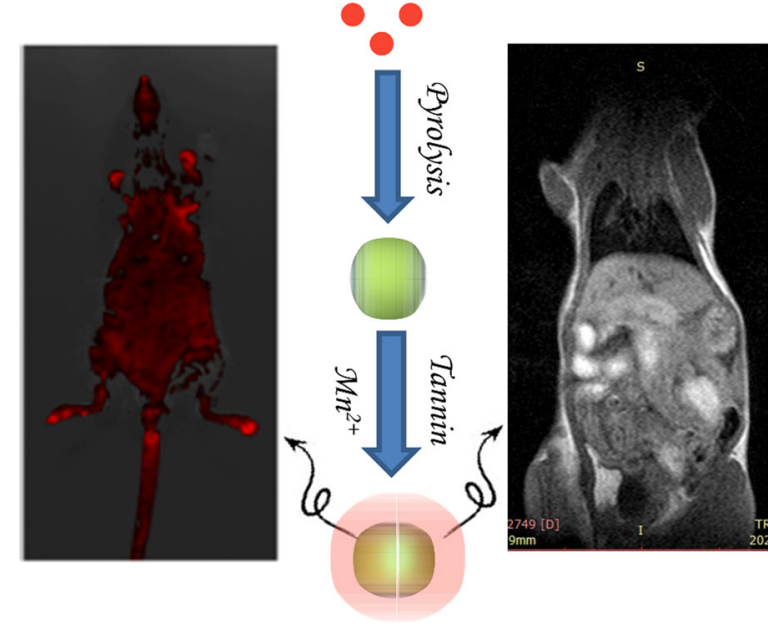

Supplementary information The online version contains supplementary material available at https://doi.org/10.1007/s10856021-06629-0.

Weibing Xu

xuwb@gsau.edu.cn

$\bowtie$ Fang Nian

nianf@gsau.edu.cn
2 College of Life Science, Gansu Agricultural University, Lanzhou 730000, China

3 Institute of Modern Physics, Chinese Academy of Sciences, Lanzhou 730000, China

1 College of Science, Gansu Agricultural University, Lanzhou 730000, China 


\section{Introduction}

The poor sensitivity restricts the application of magnetic resonance imaging (MRI) for detecting small tumor nodules [1-3]. Many methods are used to improve the sensitivity of MRI contrast agents. Among them, the introduction of fluorescence groups into the MRI contrast agents to construction of dual-model imaging probe is considered an important way, which will combine the advantages of tissue deep penetration of MRI with the advantages of high sensitivity of fluorescence imaging to overcome their shortcomings and achieve complementary advantages [4-10]. Fluorescent materials commonly used in MRI-FI dual-mode imaging probes include carbon quantum dots (CDs), organic dyes, and metal compounds. Due to the ease of surface functionalization, favorable optical properties, good biocompatibility, excellent water solubility, and low toxicity along with green synthesis, CDs are becoming a superior framework to construct MRI-FI bimodal imaging probes [11-18]. For instance, the synthesis of Gd-dopped CDs for fluorescence/MR imaging by the low temperature pyrolysis of the precursors containing $\mathrm{Gd}$ and carbon is reported $[19,20]$ The longitudinal relaxation rate ( $\mathrm{r} 1)$ and the quantum yield $(\mathrm{QY})$ of the resultant Gd-doped CDs is around 5.5-6.4 $\mathrm{mM}^{-1} \mathrm{~s}^{-1}$ and 2.6-8.9\%, respectively. However, it is well known that gadolinium ions $\left(\mathrm{Gd}^{3+}\right)$ are highly toxic because they inhibit calcium channels, induce change in intracellular reactive oxygen species levels, and cause cardiovascular and neurologic toxicity [21, 22] Unlike the lanthanides including $\mathrm{Gd}$, the non-lanthanide metal $\mathrm{Mn}$ is a natural cellular consistent, and usually acts as a cofactor for various enzymes and receptors [23]. For several years, the paramagnetic $\mathrm{Mn}(\mathrm{II})$ has been at the forefront of contrast agent research as it is believed to be one of the most promising alternatives to Gd(III) [24]. Various Mn(II) complex and composites are fabricated and to be used as MRI contrast agents [24, 25]. For instance, the Gyula Tircsó research group designed and fabricated many kinds of Mn(II)-based MRI contrast agent [26]. However, multi-step process and time-consuming are needed in the process of preparing these small molecular complexes of $\mathrm{Mn}(\mathrm{II})$. In addition, in-situ Mn-dopped CDs composites have been reported in many literatures due to the facile fabrication process [27]. However, a large number of metal ions are deeply embedded in the quantum dot, and these metal ions cannot contact the water molecules in the environment because of embedding, so they cannot play the MRI performance [28]. A small number of metal ions bind to the surface of the CDs in Mn-dopped CDs composite, thus giving rise to MRI properties. Therefore, it is believed that increasing $\mathrm{Mn}$ ions loaded on the surface of CDs is expected to improve the MRI performance of the composites. Tannic acid (TA) as a type of natural polyphenols exhibits excellent metal chelating ability. The di-galloyl groups rich in TA can serve as chelating sites for multivalent metal ions (such as $\mathrm{Mn}^{2+}$ ) thus to induce rapid selfcrosslinking of TA within minutes in water [29]. The TAmetal network could be reform on various substrates to form a conformal coating [30]. This simple and convenient particle surface functionalization technology provides good inspiration for building an MRI-fluorescent dual-mode imaging probe based on manganese ions.

Herein, the tannic acid chelated with $\mathrm{Mn}^{2+}$ ions forming shell coating on the surface of blue fluorescent emissive carbon quantum dots (BCQD) to construct novel MR/FI bimodal imaging probe. Compare to the free BCQD, the maximum excitation and emission wavelengths showed an obvious red shift phenomenon after the introduction of tannin-Mn coating on the surface of the BCQD. The content of $\mathrm{Mn}^{2+}$ is calculated via the EDS and TGA. The quantum yield of the bimodal probe (QY) is detected to be about $7.24 \%$. The structure of the as-prepared probe is verified in. detail. Afterward, the in vitro fluorescence and MR imaging are performed. The longitudinal $r 1$ value and the ratio of $r 2 /$ r1 of the probe are calculated. The metabolic pathways in vivo are inferred by studying the bio-distribution of the probe in major organs. Thus, these results indicate that probe would be an excellent dual-modal imaging probe for enhanced MR imaging and fluorescence imaging.

\section{Expermental}

\subsection{Chemicals}

Tannic acid (TA), $\mathrm{MnCl}_{2} \cdot 4 \mathrm{H}_{2} \mathrm{O}$ ( $\geq 99 \%$ ), diammonium citrate $(\geq 99 \%)$, and MOPS $(\geq 99 \%)$ were purchased from Shanghai Aladdin Bio-chem Technology Co. Ltd. DMSO $(\geq 99.5 \%)$ were purchased from Jiangsu Honghui Chemical Co. Ltd. Urea $(\geq 99 \%)$ were purchased from Shanghai Yuanye Bio-technology Co. Ltd. Ethyl acetate ( $\geq 99.5 \%)$ were purchased from Tianjin Lianlongbohua Pharmaceutical Chemistry Co., Ltd. All these chemicals were used without further purification.

\subsection{Synthesis of BCQD}

$0.75 \mathrm{~g}$ citric acid and $1.0 \mathrm{~g}$ urea (molar ratio $1: 5)$ were dispersed in $30 \mathrm{ml}$ deionized water. The mixture was transferred into $50 \mathrm{~mL}$ tetrafluoroethylene and sealed in a stainless-steel autoclave. The autoclave was heated at $180^{\circ} \mathrm{C}$ for $7 \mathrm{~h}$. After the mixture cooling to room temperature, the mixture passed through a $0.22 \mu \mathrm{m}$ injection filter to remove large lumps. Then, it was further purified by dialysis with dialysis bag $\left(\mathrm{M}_{\mathrm{w}}=3500\right)$ for 48 hours, and the solid samples were obtained by freeze drying and it was named BCQD. 


\subsection{Synthesis of BCQD@Mn composite}

The $50 \mathrm{mg}$ sample was dissolved in $4.9 \mathrm{~mL}$ of deionized water with the assistance of ultrasound. Then, $50 \mu \mathrm{l}$ $\mathrm{MnCl}_{2} \cdot 4 \mathrm{H}_{2} \mathrm{O}, 50 \mu \mathrm{l}$ tannic acid (TA) were added into the above solution (final concentration: $\mathrm{MnCl}_{2} \cdot 4 \mathrm{H}_{2} \mathrm{O}: 0.1 \mathrm{mg}$ / $\mathrm{ml}$, TA: $0.4 \mathrm{mg} / \mathrm{ml}$, BCQD: $10 \mathrm{mg} / \mathrm{mL}$ ). The solution was vigorously mixed by a vortex mixer for $10 \mathrm{~s}$ immediately. The $\mathrm{pH}$ of this solution was subsequently raised by adding $0.5 \mathrm{ml}$ of MOPS buffer ( $20 \mathrm{mM}, \mathrm{pH}$ 7.4). The excess TA and $\mathrm{MnCl}_{2}$ was removed by dialysis for $48 \mathrm{~h}$. The solution after dialysis was lyophilized to obtain a black powder, named BCQD@Mn. The typical fabrication process was exhibited in Scheme 1.

\subsection{Characterization}

Using Bruker IFS66v/s IR spectrometer (Bruker, Karlsruhe, Germany) was used to analyze the function group of the sample in the range of $400-4,000 \mathrm{~cm}^{-1}$ with the resolution of $4 \mathrm{~cm}^{-1}$. The morphologies of BCQD@Mn were characterized using a JEM-1200 EX/S transmission electron microscopy (TEM) (JEOL, Tokyo, Japan) and an S-4800 field emission scanning electron microscopy (SEM) (HITACHI, Tokyo, Japan). BCQD@Mn was measured by TU-1901 dual-beam UV-vis spectrophotometer. The surface elements were measured by X-ray photoelectron spectroscopy (XPS) with VG ESCALAB220i-XL spectrometer.

\subsection{Quantum yield}

The fluorescence quantum yield $(\varphi)$ of BCQD@Mn was calculated by using Quinine sulfate solution as a standard. Quinine dissolved in $0.5 \mathrm{M} \mathrm{H}_{2} \mathrm{SO}_{4}$ was used as a standard with a known $\varphi$ value of $54 \%$ at $345 \mathrm{~nm}$ excitation wavelength. The $\varphi$ of BCQD@Mn was calculated by comparing the UV-vis absorbance values and the integrated fluorescence intensities $\left(\lambda_{\mathrm{ex}}=360 \mathrm{~nm}\right)$ of the BCQD@Mn with those of quinine sulfate solution. The absorbance values of all solutions were maintained under 0.05 to minimize selfabsorption. Eq. 1 was used for the calculation of $\varphi$ of BCQD@Mn.

$$
\varphi=\varphi_{s} \times \frac{I}{I_{s}} \times \frac{A_{s}}{A} \times \frac{\eta^{2}}{\eta_{s}^{2}}
$$

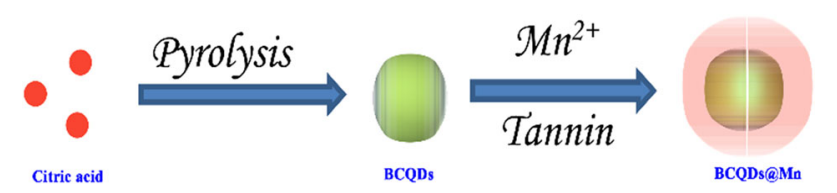

Scheme 1 Schematic illustration for fabrication of BCQD@Mn composite where $\varphi$ is fluorescence quantum yield. I is the integrated fluorescence intensity, A is the UV-Vis absorbance, and $\eta$ is the refractive index of the solvent for BCQD@Mn suspension solution as water $(\eta=1.33)$ and $0.5 \mathrm{M} \mathrm{H}_{2} \mathrm{SO}_{4}$ in water $(\eta=1.76)$.

\subsection{Cytotoxicity assay}

Typically, HepG2 cells $\left(5 \times 10^{3}\right.$ cells/well) were seeded into a 96-well plate and further incubated in RPMI1640 containing FBS $(10 \%)$ at $37{ }^{\circ} \mathrm{C}$ for $24 \mathrm{~h}$. Following overnight incubation, the BCQD@Mn composite was added at different concentrations for $48 \mathrm{~h}$. After incubation, the MTT reagent $(20 \mu \mathrm{L}, 5 \mathrm{mg} / \mathrm{ml})$ was added into each well of the 96-well culture plate, and cells were incubated for another $4 \mathrm{~h}$ at $37{ }^{\circ} \mathrm{C}$ in an atmosphere of $5 \% \mathrm{CO}_{2}$. Then the medium was taken out and $150 \mu \mathrm{L}$ DMSO was added to each well. Cell viability was assessed by the optical density (OD) value at a wavelength of $490 \mathrm{~nm}$ using an enzyme-labeled instrument.

\subsection{In vitro and vivo fluorescence imaging}

The HepG2 cells $\left(2 \times 10^{5}\right.$ cells/well $)$ were seeded into sixwell plates, cultured for $24 \mathrm{~h}$, and treated with BCQD@Mn composite for $48 \mathrm{~h}$. After treatment, the culture medium was discarded, and cells were washed three times with PBS. Then the cells were washed three times with PBS for $7.0 \mathrm{~min}$ each time and imaged by fluorescence microscope. In vivo fluorescence imaging experiments were conducted in Ultra-sensitive fluorescence imaging system for small animals (VISQUE In-vivo Smart-LF, Viewers). The BCQD@Mn composite was dissolved in PBS at a concentration of $20 \mathrm{mg} / \mathrm{ml}$. Healthy male Kunming mice (Experimental Animal Canter of Lanzhou University, $20 \pm$ $2 \mathrm{~g})$ were randomly divided into two groups $(n=3)$ and were given of the BCQD@Mn composite one time via tail vein $(20 \mu \mathrm{L}$ injection volume, $20 \mathrm{~g} / \mathrm{kg})$, control group received PBS. All animals were sacrificed after seven days. Major organs (Heart, liver, spleen, lung, kidneys) were removed, washed with PBS, and fixed in $4 \%$ formaldehyde, embedded in paraffin, sectioned, and then stained with hematoxylin and eosin for histological analysis. Mice were intravenously injected with the composite solution $\left(10 \mathrm{mg} \cdot \mathrm{kg}^{-1}\right)$ and fluorescence imaging was conducted at $0.5,1,2,4$, and $24 \mathrm{~h}$ post injection. Ex vivo fluorescence imaging of major organs was also conducted.

\subsection{In vitro and in vivo MRI performance}

In vitro MRI experiments were conducted in a $0.5 \mathrm{~T}$ nuclear magnetic resonance analyzer (MesoMR23-060H-I, Suzhou Niumag Analytical Instrument Co., China). The MRI 
samples were prepared by drawing BCQD@Mn composite solutions at different $\mathrm{Mn}$ (II) concentrations into $1.0 \mathrm{~mL}$ disposable poly (propylene) syringes. For the T1 measurement, inversion-recovery fast spin-echo sequence with eight inversion times (TI; ranging from $10 \mathrm{~ms}$ to $4 \mathrm{~s}$ ) was employed. Meanwhile, for the T2 measurement, multiclade multi-echo sequence was employed. [Others parameters: $\mathrm{TR}=6 \mathrm{~s}$ (for T1) and $2 \mathrm{~s}$ (for T2); matrix size $=128 \times 128$; field of view $=40 \times 40 \mathrm{~mm}$; slice thickness $=4 \mathrm{~mm}$ ). The $\mathrm{r} 1$ and $\mathrm{r} 2$ values were calculated from the slop of curvefitting result of $1 / \mathrm{T} 1$ and $1 / \mathrm{T} 2\left(\mathrm{~s}^{-1}\right)$ versus the $\mathrm{Mn}(\mathrm{II})$ concentration $(\mathrm{mM})$. for in vivo MRI, healthy male Kunming mice $(20 \pm 2 \mathrm{~g}$,) were also randomly divided into two groups $(n=3)$. The animal was anesthetized using chloral hydrate $(10 \mathrm{wt} \%, 80 \mu \mathrm{L}) .20 \mu \mathrm{L}$ of the $\mathrm{Mn}(\mathrm{II})$ composite $\left(40 \mu \mathrm{g} \mathrm{mL}^{-1}\right)$ solution was injected into the animal one time via the tail vein. MRI was performed at the pre-injection and $0.5,1.0,4.0$, and $24 \mathrm{~h}$ after injection. The T1-weighted coronal MRI was acquired using a MesoMR23-060H-I 0.5 T MRI scanner with the following imaging parameters: repetition time, $330 \mathrm{~ms}$; echo time, $18.2 \mathrm{~ms}$; field of view, $100 \times 100 \mathrm{~mm}^{2}$; and slice thickness, $3.0 \mathrm{~mm}$.

\section{Results}

\subsection{Structural characterization}

The detailed preparation process is described in Scheme 1 . The preparation of the composite underwent two steps including pyrolysis and coating. The UV-Vis absorption curves of BCQD and BCQD@Mn samples are displayed in Fig. S1a. Obvious absorption peaks in the near-ultraviolet region of 190-210 $\mathrm{nm}$ can be observed for the BCQD. As for the BCQD@Mn, two significant peaks are found and the peak's center locates at 210 and $280 \mathrm{~nm}$, respectively. The fluorescence emission spectra of BCQD at different excitation wavelengths and concentrations are exhibited in Fig. $\mathrm{S} 1 \mathrm{~b}$, c. The relative intensity increases first and then decreases with the increase of excitation wavelength. The maximum excitation and emission wavelength is 350 and $450 \mathrm{~nm}$, respectively. In addition, a good dependence relationship is observed between the intensity and concentration. The relationship between excitation wavelength and concentration and emission intensity of BCQD@Mn composite is like that of the BCQD (Fig. S1d, e). The maximum excitation and emission wavelengths of the BCQD@Mn composite is observed at $390 \mathrm{~nm}$ and $490 \mathrm{~nm}$ (Fig. S1f), respectively. The fluorescence quantum yields in PBS are determined to be $7.24 \%$ according to the corresponding formula and quinine sulfates are used as a reference [31]. The FTIR spectrum of the BCQD and BCQD@Mn complex is shown in Fig. 1a. A broad absorption band can be seen at 3400 and $1740 \mathrm{~cm}^{-1}$ for both samples, which is assigned to the characteristic stretching vibration of $-\mathrm{OH}$ and $\mathrm{C}=\mathrm{O}$ groups $[32,33]$. The peaks located at 757 and $1314 \mathrm{~cm}^{-1}$ can be ascribed to the stretching vibration of $\mathrm{C}-\mathrm{H}$ in both samples. The characteristic peak at 1030, 1082, and $1189 \mathrm{~cm}^{-1}$ can be assigned to the stretching vibration of $\mathrm{C}-\mathrm{O}$ in both samples [34]. The TGA and DTG curves of the BCQD and BCQD@Mn complex are presented in Fig. 1b. Three weight loss steps are found for the BCQD. The first one located in the temperature range of $100-400{ }^{\circ} \mathrm{C}$ is assigned to the oxidation of functional groups in the sample [35]. The second sharp process of weight loss between 400 and $600{ }^{\circ} \mathrm{C}$ is ascribed to the carbonization of skeletons in the BCQD [36]. The third bluff type of weightlessness is in the temperature range of 600 to $700{ }^{\circ} \mathrm{C}$. There are three exothermic peaks on the DTG curve, and the largest one is between 600 and $700{ }^{\circ} \mathrm{C}$. For the BCQD@Mn, the weightlessness process is basically similar to that of the BCQD. The XRD patterns of both samples showed a diffraction peak at $22.5^{\circ}$, which is attributed to the graphitized structure of carbon. The intensity decreases after the introduction of TA-Mn coating on the surface of BCQD. This may be related to reducing the crystallinity without affecting its structure. The morphology of BCQD@Mn composite is studied by TEM (Fig. 1d). Many nanoparticles with regular spherical structures have been observed, which are cross-linked with each other due to agglomeration. Meanwhile, the size is about $20 \mathrm{~nm}$ according to the statistics. This indicates that the structure of the BCQD is kept very well in the process of introducing TA-Mn coating. In addition, DLS is also used to monitor the preparation of the BCQD@Mn (Fig. 2a). It can be found that the average hydrodynamic diameter is calculated to be $64.41 \pm$ $16.67 \mathrm{~nm}$ from a DLS pattern. The PDI value is as low as 0.003 , indicating that the high granular uniformity of the composite. The value of D10, D50, and D90 is around $28.68 \mathrm{~nm}, 41.54 \mathrm{~nm}$, and $65.76 \mathrm{~nm}$, respectively. This means that the average size is less than $28.68 \mathrm{~nm}, 41.54 \mathrm{~nm}$ and $65.76 \mathrm{~nm}$ particles account for $10 \%, 50 \%$, and $90 \%$ of all particles. This value is larger than the average size estimated from TEM. This may be ascribed to a large number of hydrated water molecules around the composite NPs in the DLS test procession. The successful formation of BCQD@Mn is further verified by XPS spectra. The survey spectrum confirmed that BCQD@Mn is mainly composed of $\mathrm{C}, \mathrm{N}, \mathrm{O}$, and $\mathrm{Mn}$ (Fig. 2b). The corresponding characteristic peaks for $\mathrm{C} 1 \mathrm{~s}, \mathrm{~N} 1 \mathrm{~s}, \mathrm{O} 1 \mathrm{~s}$, and $\mathrm{Mn} 2 \mathrm{p}$ are observed at 285.6, 399.32, 531.9, and $640 \mathrm{eV}$, respectively. Moreover, the content of all elements from the XPS results is summarized and inserted in Fig. 2b. As expected, XPS results confirmed the presence of $\mathrm{Mn}$ in the product with 0.34 At\% (Table S1). The high-resolution spectra of Mn 2p 
Fig. 1 IR spectrum (a), TGA and DTG curves (b) and XRD pattern (c) of BCQD and BCQD@Mn; (d) TME image of the BCQD@Mn composite
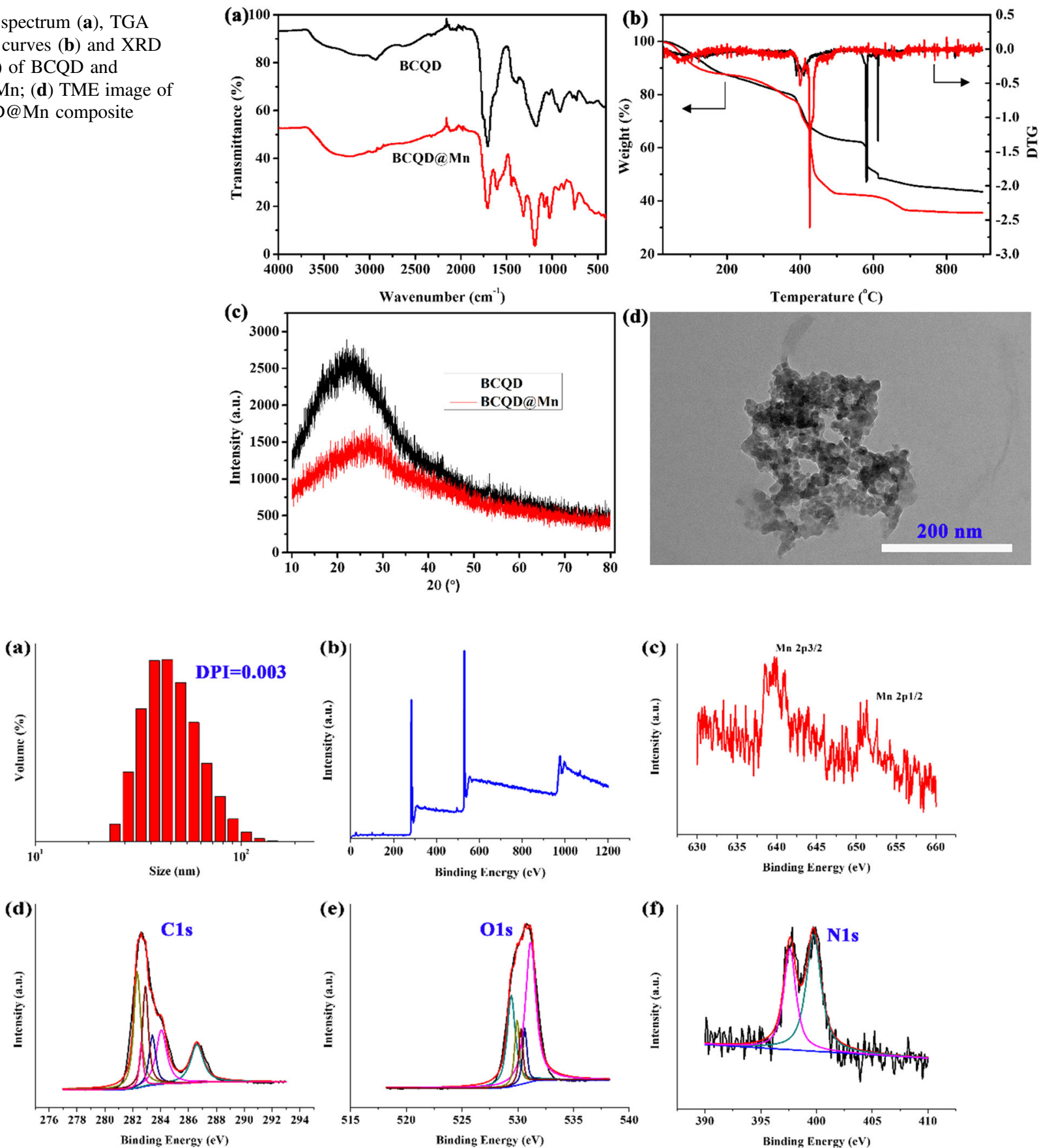

Fig. 2 DLS curve (a) and XPS spectra of the BCQDs@Mn composite: (b) survey spectra, (c) Mn 2p, (d) C 1s, (e) O 1s, (f) N 1s

(Fig. 2c) present two peaks at 652.6 and $642.1 \mathrm{eV}$, which agreed with the spectra of Mn (2p1/2) and Mn (2p3/2) [37]. The high-resolution XPS spectra of C 1s (Fig. 2d) revealed the presence of $\mathrm{sp} 3$ and $\mathrm{sp} 2$ hybridized carbon atoms and carboxylate/hydroxyl moieties in the composite [38]. The fitted peaks in $\mathrm{N} 1 \mathrm{~s}$ spectra suggest the $\mathrm{C}-\mathrm{N}, \mathrm{C}=\mathrm{N}$, and graphitic- $\mathrm{N}$ content are involved in the composite. As for $\mathrm{O}$ $1 \mathrm{~s}$, the fitted peaks correspond to $\mathrm{C}-\mathrm{O}$ and $\mathrm{C}=\mathrm{O}$. The energy-dispersive X-ray spectroscopy (EDS) analysis demonstrated the distribution of $\mathrm{Mn}, \mathrm{O}, \mathrm{C}$, and $\mathrm{N}$ elements in the BCQD@Mn composite (Fig. S2a). The relative content in mass percentage of the $\mathrm{C}, \mathrm{N}, \mathrm{O}$, and $\mathrm{Mn}$ is around $67.71 \%, 3.87 \%, 27.48 \%$, and 0.94 At \% (Table S2), respectively. The elemental mapping (Fig. S2b-f) revealed the four elements are uniformly distributed in the composite. This result implied that the TA-Mn coating is fully confined within the surface of BCQD during the reaction process, and all components are strongly coupled together 
in the composite. The stability of the BCQD and BCQD@Mn composite is inspected via the change in particle size and potential with immersion time (Fig. S3a, b). The average particle sizes and PDI of BCQD and BCQD@Mn composite is detected to be $120 \mathrm{~nm}, 150 \mathrm{~nm}$, and $0.461,0.409$ on the first day. Both numerical of the BCQD@Mn composite increase up to $163 \mathrm{~nm}$ and 0.507 as the time extends to fifth day. Although the size of BCQD shows insignificant change over time, the PDI value increased by 0.523 . The slight trend of both values even immersed in PBS solution for $120 \mathrm{~h}$ in PBS indicate good stability performance of both samples. The Zeta potential values of BCQD@Mn composite are -15.4 and $-16.3 \mathrm{mV}$ for the first and fifth day, respectively. This value is significantly higher than -25.3 and $-26.1 \mathrm{mV}$ of BCQD at the same time. this may be related to the introduction of the TAMn layer on the surface of the BCQD. This further reflects the excellent stability of the BCQD@Mn composite in solution state. The content of $\mathrm{Mn}$ in the BQCD@Mn is further estimated via the atomic absorption spectrum. Firstly, a calibration curve is established by using the function relationship between the concentration of different manganese ions and the absorption intensity (Fig. S3c). A good linear correlation can be found between intensity and the concentration of $\mathrm{Mn}^{2+}$ with a correlation coefficient of $\mathrm{R}^{2}=0.99944$. The BCQD@Mn $(1.0 \mathrm{mg})$ is formulated into a $10 \mathrm{~mL}$ solution in deionized water. The content of $\mathrm{Mn}$ in the composite is calculated by comparing the absorption intensity of the above solution to the calibration curve. The content of $\mathrm{Mn}$ is about $0.22 \mathrm{mM}$ in the BCQD@Mn composite solution.

\subsection{Cytotoxicity and fluorescence bioimaging}

It is believed that low toxicity is emerging as the focus of designing metal complexes for the diagnosis of cancers. MTT assay is employed to evaluate the cytotoxicity of BCQD@Mn composite toward HepG2 cells. As shown in Fig. S3d, the cell viability is over $90 \%$ even when the concentration of BCQD@Mn reaches $100 \mu \mathrm{g} \mathrm{mL}^{-1}$ (equivalent $\mathrm{Mn}$ concentration is $0.06 \mathrm{mM}$ ) for $48 \mathrm{~h}$ incubation, indicating the safety and negligible cytotoxicity of the composite towards HepG2 cells. This may be ascribed to the strong Mn-TA chelation effectively restraints the leakage of $\mathrm{Mn}^{2+}$ into the surroundings. The feasibility of the BCQD@Mn composite for in vitro luminescent cell imaging in HepG2 cancer cells after 24 h-incubation is conducted by fluorescence microscope. As illustrated in Fig. S4a, d, g, the bright-field image of BCQD@Mn with different amount demonstrated the morphological integrity of cells, validating their biocompatibility. The fluorescence of the cells is distinctly enhanced with the amount of BCQD@Mn (Fig. S4b, e, h), suggesting a dosagedependent fluorescence enhancement relationship. It should be noted that the BCQD@Mn are observed to localize in the whole cell area, weak emission signals from the nucleus and strong ones from the cytoplasm (Fig. S4c, f, i), indicating excellent cell membrane permeability. The tubes fluorescence images of BCQD@Mn composite solutions are obtained under four different excitation wavelengths (Fig. S5a-d). The solution only exhibits good fluorescence properties at the excitation wavelength of $390-490 \mathrm{~nm}$. Afterward, the nanoplatform as a in vivo fluorescent imaging agent is explored. The whole-body fluorescence images of mice are taken at different time points after the tail vein injection of BCQD@Mn composite (Fig. 3). It can be found that the fluorescence of BCQD@Mn composite could efficiently penetrate mice skin and tissues. The mice injected with PBS exhibits the weakest fluorescence, which is derived from the spontaneous fluorescence of mice under the selected excitation wavelength. No animal showed any signs of acute toxicological responses during the experiments. In order to further explore the metabolic pathway of the probe, fluorescence imaging of the main organs at different time points is performed (Fig. 4). The mice are sacrificed at different time points $(24,4,2,1$, and $0.5 \mathrm{~h})$ to obtain organs imaging at the same time. The fluorescence intensity of the same dissected organ at different time points is gradually reduced, this is agreeing well with the wholebody fluorescence imaging. Besides, fluorescence signals of the brain, liver, and kidney are always much stronger than these of heart, spleen, and lung. Surprisingly, significant fluorescent signals are observed in the brains of the mice at the $0.5,1$, and $2 \mathrm{~h}$, respectively, suggesting the potential of the BCQD@Mn composite to cross the blood-brain barrier, which may provide a valuable strategy for the theragnostic
Fig. 3 In vivo whole-body fluorescence images of mice with intravenous injection of the BCQD@Mn at different time points $(n=3)$
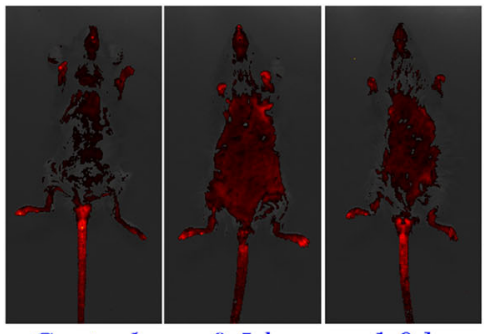

Control

$0.5 h$

$1.0 \mathrm{~h}$
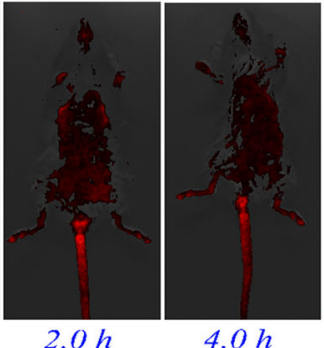

$4.0 h$
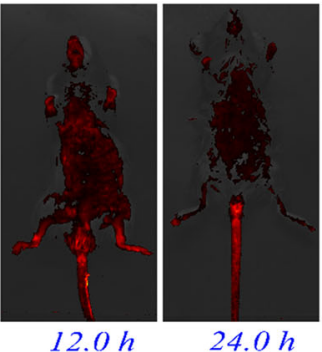
Fig. 4 Real-time ex vivo imaging of mice with intravenous injection of the BCQD@Mn composite at different time points $(n=3)$

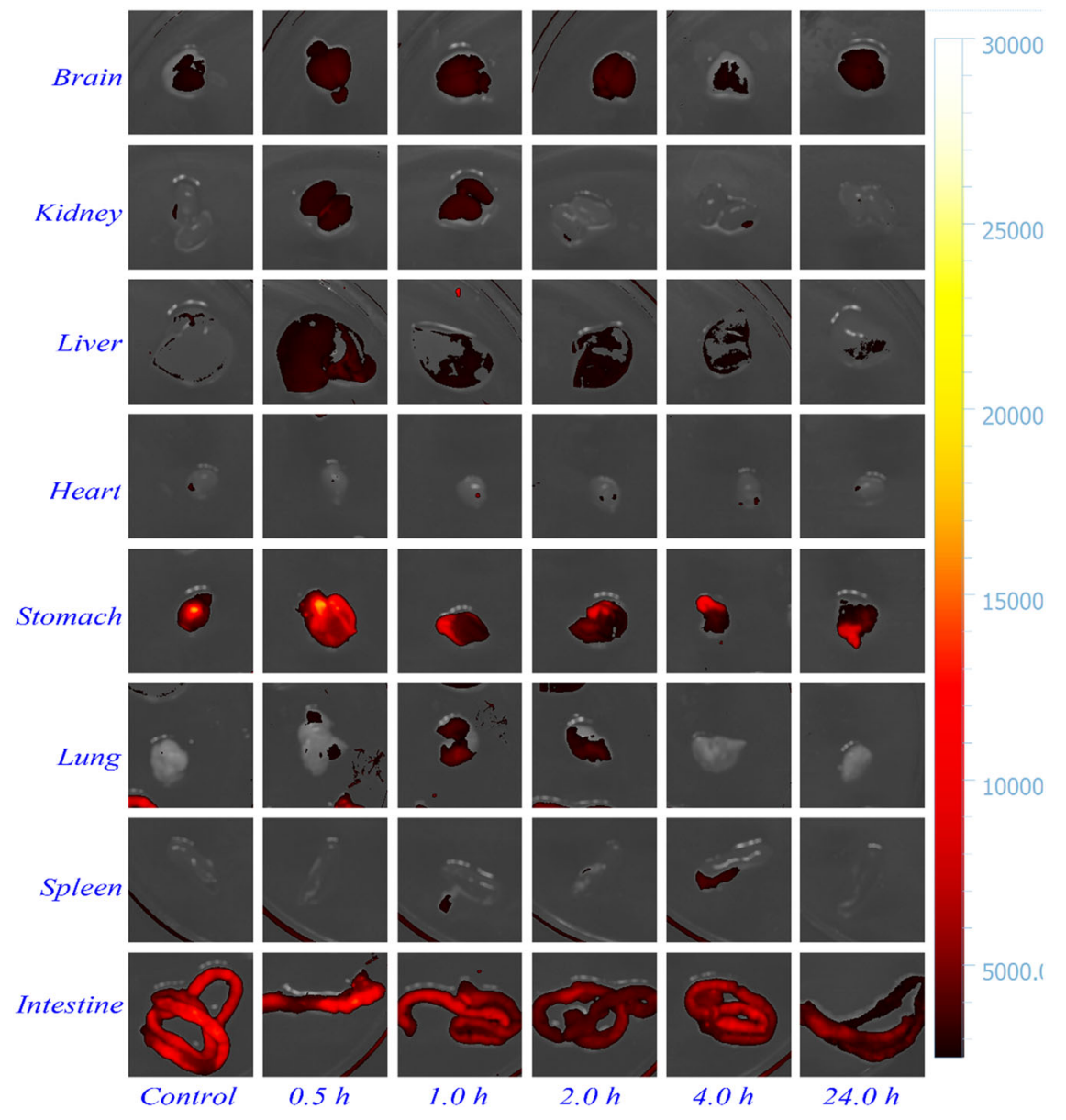

is investigated. The T1 and T2 MRI images are illuminated in Fig. 5a. Although the brightness of both $\mathrm{T} 1$ and $\mathrm{T} 2$ images increases with the increase of $\mathrm{Mn}$ (II) concentration, the improvement degree of $\mathrm{T} 1$ images is significantly higher than that of $\mathrm{T} 2$ images, indicating the composite gives clearly enhanced MR signals in the in vitro imaging experiments. The relaxivity $\mathrm{r} 1$ and $\mathrm{r} 2$ derived from the concentration-dependent $\mathrm{T} 1$ and $\mathrm{T} 2$ measurement is found to be 2.43 and $10.82 \mathrm{mM}^{-1} \mathrm{~S}^{-1}$ (Fig. 5b), respectively. These results suggest the BCQD@Mn composite could be a good probe for MRI CAs. In vivo, MRI is investigated in normal mice. The time-dependent T1-weighted 2D coronal images of pre- and post injection of BCQD@Mn composite at various time points are shown in Fig. 6. The particles are intravenously injected into the animals. It is clearly observed that the T1 MR signal in the liver and kidneys increases significantly within the $1 \mathrm{~h}$ post injection and then decreases as the time further extension. However, the T1 MR signal in the intestine organ gradually increased within $8 \mathrm{~h}$, and then turned weaker over time. After $24 \mathrm{~h}$, signals in most of the organs had subsided to the pre-injection levels, indicating excretion of the particles from the circulation. Interestingly, the signal change in the bladder is found to be
Manganese ion (Mn(II)) has been proved to be a safe paramagnetic ion, and MRI contrast agents based on Mn (II) has attracted extensive attention for many years. The performance of BCQD@Mn composite as potential MRI CAs 

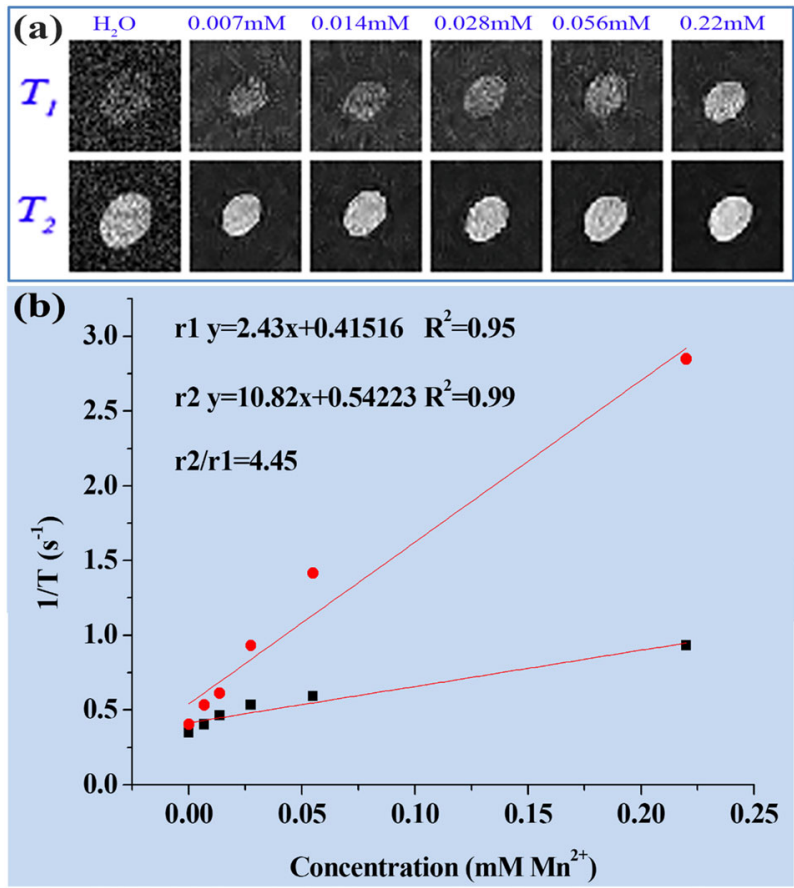

Fig. 5 a In vitro T1 and T2-weighted MRI images, (b) r1 and r2 values of the BCQD@Mn composite at different time points

small throughout the course of the experiment. Instead, there is a dramatic increase of signals in the liver and intestinal, indicating the liver metabolism is the main metabolic pathway of the BCQD@Mn composite.

\section{Discussion}

During the current decades, the great numerous of Fl-MR dual-mode imaging probs based on $\mathrm{Gd}^{3+}$ has been investigated [39]. Nevertheless, most of the currently approved contrast agents based on $\mathrm{Gd}^{3+}$ possess long-term toxicity for living imaging. To overcome this problem, the BCQD@Mn nanocomposite is designed with a two-step approach. BCQD are firstly synthesized by one-pot pyrolysis method, and Mn-TA films are coated onto BCQD with rapid layer by layer interactions between TA and MN (II) ions.

The successful synthesis of the BCQD@Mn nanocomposites is proved by several evidences. As shown in the UV-Vis spectra in Fig. S1a, a new peak is found at $280 \mathrm{~nm}$ for the BCQD@Mn nanocomposite, indicating the existence of $\mathrm{p}-\pi^{*}$ and $\mathrm{n}-\pi^{*}$ transition in the nanocomposite. This may be attributable to the existence of $\mathrm{sp}^{2}$ hybridized carbon atoms [31], suggesting the introduction of the TA$\mathrm{Mn}$ coordination polymer coating on the surface of the BCQD successfully. Similarly, the maximum excitation and emission wavelengths in the fluorescence spectrum (Fig. S1f) show an obvious red shift phenomenon, reaching
$390 \mathrm{~nm}$ and $490 \mathrm{~nm}$ after the surface of the BCQD is coated by tannin-Mn layer. This may be due to the introduction of coordination polymer layers to increase the conjugated structure of the system. In the FTIR spectrum (Fig. 1a), the absorption band at about 1605 and $1442 \mathrm{~cm}^{-1}$ in the BCQD@Mn complex is ascribed to the characteristic of the stretching of the benzene ring of TA [40]. This suggests that TA-Mn coordination polymer is successfully introduced on the surface of the BCQD. As for the TGA process (Fig. 1b), the maximum weightlessness process of the BCQD@Mn composite occurs between 400 and $600{ }^{\circ} \mathrm{C}$, which is completely different from the range for BCQD. This may be related to the introduction of TA-Mn coating. Furthermore, the XRD peaks of the synthesized BCQD@Mn nanocomposites are consistent with BCQD (Fig. 1c), and the peaks exhibit slight broadening and uneven peak profiles due to the thick TA-Mn films out of the BCQD cores [41]. The films surrounding the BCQD@Mn nanocomposites NPs can be easily identified in the TEM images (Fig. 1d). The EDS and XPS results (Fig. S2 and Fig. 2b-f) also indicate that $\mathrm{Mn}$ (II) has cooperated into this nanocomposite (Tables S1, S2). The test value of EDS is greater than XPS for Mn element, which is related to the XPS can only obtain the surface element content of nanoparticles [42]. Together with the above characterizing result, Mn-TA films are confirmed to be formed through complexation between tannic acid molecule and Mn (II). More importantly, MPN films obviously enhance the biocompatibility and lower cytotoxicity (Fig. S3b) due to the water solubility of tannic acid molecule.

Afterward, the MR/FI bi-modalities imaging performance of the BCQD@Mn nanocomposite is synergistically investigated. The CLSM observes that the HepG2 cells incubated with the nanocomposite show green fluorescence imaging (Fig. S4). The clear and important information about cell morphology and physiological characteristics can be obtained from the visible cell imaging. The MRI property of the as-prepared BCQD@Mn nanocomposite is mainly due to the five unpaired inner electrons of $\mathrm{Mn}$ (II) [43]. T1 and T2 weighted images (Fig. 5a) reflect the efficiency of a MRI contrast agent, and the r1 value of BCQD@Mn nanocomposite in vitro is $2.43 \mathrm{mM}^{-1} \mathrm{~s}^{-1}$ (Fig. 5b). In other word, this reflects that the BCQD@Mn nanocomposite can be served as bio-marker. On this basis, the in, ex vivo fluorescence imaging and in vivo MRI is exhibited in Figs. 3, 4 and 6 . It can be seen that the fluorescence is stronger than that of the control group at all post injection time points even $24 \mathrm{~h}$. Not surprisingly, the fluorescence intensity gradually slight decreases as the extension of time. This suggests that the complex has a long residence time in vivo, which would facilitate multiple iterations of imaging after a single injection. However, fluorescence imaging is mainly limited by two aspects: on the one hand, the tissue penetration ability of 
Fig. 6 In vivo T1-weighted MRI images of mice with intravenous injection of the BCQD@Mn composite at different time points
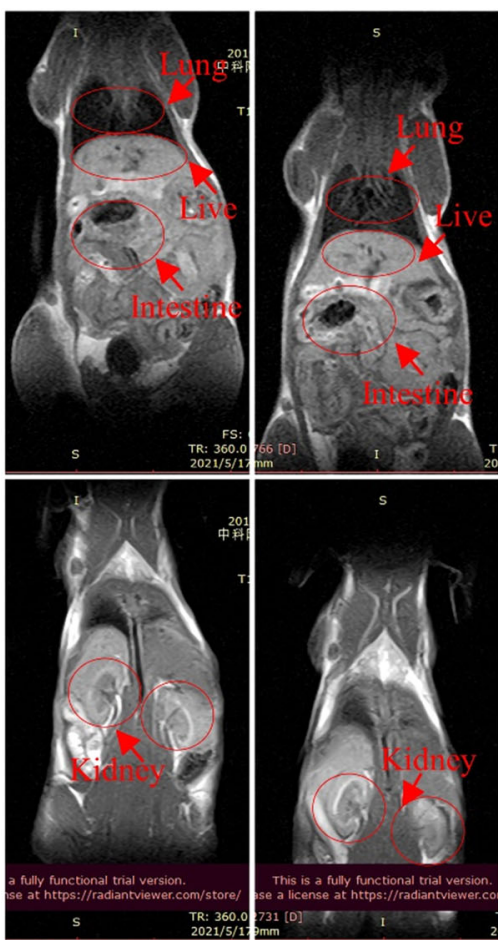

pre-injection

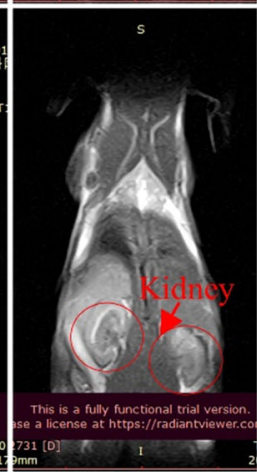

$1.0 \mathrm{~h}$
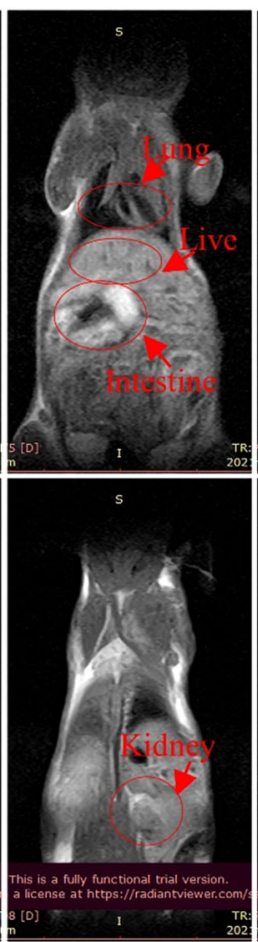

4.0 h
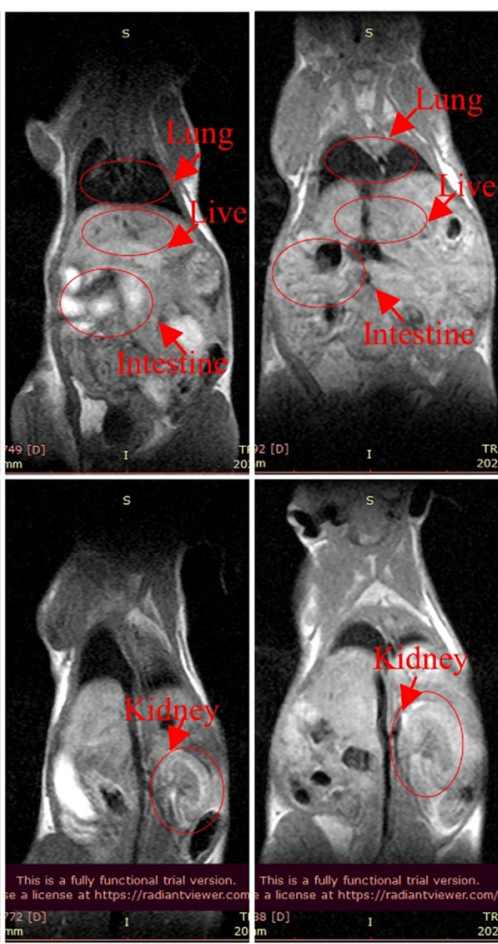

8.0 h

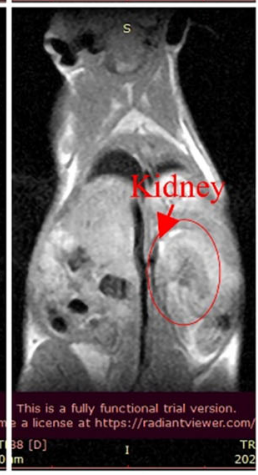

20.0 h fluorescence is weak, the fluorescence agents in vivo are difficult to be excited, and the emitted fluorescence is also difficult to be detected. On the other hand, spontaneous fluorescence from biological tissue can cause serious interference to the fluorescence of probes [44]. In view of the possible background interference of whole-body fluorescence signals, the fluorescence signals of ex vivo organs tissues with whole-body MRI signals are compared in Figs. 4 and 6 . It can be found that fluorescence signals in the kidneys and liver reaches the highest levels at $1 \mathrm{~h}$ of post injection, while MRI signals in both organs improve significantly at the same post injection time compare to the control. Although the fluorescence signals in the liver and kidneys decreased significantly at $1 \mathrm{~h}$ of post injection, the signal fluorescence signals in the intestine began to increase significantly from this point on, this indicates that the compound enters the small intestine rapid metabolism through the liver and kidneys. Correspondingly, the exact signal change relationships could be found in intestine T1 MR signal. Figure 6 shows that the in vivo intestine T1 MR signal gradually increased within $8 \mathrm{~h}$ post injection. The complementary of fluorescence signals from ex vivo organs and in vivo MRI signals shows that there are two metabolic pathways included in liver and kidneys of the composites in mice. The above results showed that BCQD@Mn nanocomposites possess an excellent MRI and fluorescence di-model imaging probe in vivo. This is very useful for obtaining comprehensive diagnostic information from different images of the same tissue or organ.

\section{Conclusions}

In conclusion, TA-Mn coordination polymer is introduced onto the surface of BCQD by a convenient in-situ synthesis method to successfully fabricate novel MRI-FL dimodel image probe. Instead of damaging the fluorescence of quantum dots, the introduction of TA-Mn coating redshifts the emission wavelengths of CQDs, which is more conducive to fluorescence imaging. Interestingly, the BCQD@Mn(II) shows excellent bright orange fluorescence properties with the QY 7.24\%, as well as paramagnetic nature with the values of $\mathrm{r} 1$ and $\mathrm{r} 2$ is about 2.43 and $10.82 \mathrm{mM}^{-1} \mathrm{~S}^{-1}$. Real-time fluorescence imaging in vivo shows that the probe can easily enter the body of mice and gradually attenuated with time. Fluorescence imaging of the ex vivo organ revealed that the probe has two metabolic pathways in the body, the liver, and the kidneys. The results indicate that probe will be an excellent dual-modal imaging probe for enhanced MR imaging and fluorescence imaging.

Acknowledgements The authors acknowledge the support of Natural Science Foundation of Gansu Province (No: 20JR10RA511, 2019B084) and Scientific Research start-up funds for openly recruited doctors of Gansu Agricultural University (No. 2017RCZX-42).

\section{Compliance with ethical standards}

Conflict of interest The authors declare no competing interests. 
Publisher's note Springer Nature remains neutral with regard to jurisdictional claims in published maps and institutional affiliations.

Open Access This article is licensed under a Creative Commons Attribution 4.0 International License, which permits use, sharing, adaptation, distribution and reproduction in any medium or format, as long as you give appropriate credit to the original author(s) and the source, provide a link to the Creative Commons license, and indicate if changes were made. The images or other third party material in this article are included in the article's Creative Commons license, unless indicated otherwise in a credit line to the material. If material is not included in the article's Creative Commons license and your intended use is not permitted by statutory regulation or exceeds the permitted use, you will need to obtain permission directly from the copyright holder. To view a copy of this license, visit http://creativecommons. org/licenses/by/4.0/.

\section{References}

1. Angelovski G. What we can really do with bioresponsive MRI contrast agents. Angew Chem Int Ed. 2016;55:7038-46.

2. Sun X, Zhang MZ, Du RH, Zheng XJ, Tang CG, Wu YQ, He JC, Huang W, Wang YY, Zhang ZY, Han XL, Qian JC, Zhong K, Tian XH, Wu LF, Zhang GL, Wu ZY, Zou DH. A polyethyleneimine-driven self-assembled nanoplatform for fluorescence and MR dual-mode imaging guided cancer chemotherapy. Chem Eng J. 2018;350:69-78.

3. Han CP, Xie T, Wang KY, Jin S, Li K, Dou PP, Yu NN, Xu K. Development of fluorescence/mr dual-modal manganesenitrogendoped carbon nanosheets as an efficient contrast agent for targeted ovarian carcinoma imaging. J Nanobiotechnol. 2020;18:175.

4. Xiao LS, Mertens M, Wortmann L, Kremer S, Valldor M, Lammers T, Kiessling F, Mathur S. Enhanced in vitro and in vivo cellular imaging with green tea coated water-soluble iron oxide nanocrystals. ACS Appl Mater Interfaces. 2015;7:6530-40.

5. Chen HT, Wang Y, Wang T, Shi DX, Sun ZR, Xia CH, Wang BQ. Application prospective of nanoprobes with MRI and FI dualmodality imaging on breast cancer stem cells in tumor. J Nanobiotech. 2016;14:52-61.

6. Lartigue L, Coupeau M, Lesault M. Luminophore and magnetic multicore nanoassemblies for dual-mode MRI and fluorescence imaging. Nanomaterials. 2020;10:28-39.

7. Zheng SH, Zhang M, Bai HY, He MJ, Dong LN, Cai LL, Zhao MM, Wang Q, Xu K, Li JJ. Preparation of AS1411 aptamer modified Mn-MoS2 QDs for targeted MR imaging and fluorescence labelling of renal cell carcinoma. Int J Nanomed. 2019;14:9513-24.

8. Wang H, Shen J, Li YY, Wei ZY, Cao GX, Gai Z, Hong KL, Banerjee P, Zhou SQ. Magnetic iron oxide-fluorescent carbon dots integrated nanoparticles for dual-modal imaging, nearinfrared light-responsive drug carrier and photothermal therapy. Biomater Sci. 2014;2:915-23.

9. Li H, Parigi G, Luchinat C, Meade TJ. Bimodal fluorescencemagnetic resonance contrast agent for apoptosis imaging. $\mathrm{J}$ Am Chem Soc. 2019;141:6224-33.

10. Yan RQ, Hu YX, Liu F, Wei SX, Fang DQ, Shuhendler AJ, Liu $\mathrm{H}$, Chen HY, Ye DJ. Activatable NIR fluorescence/MRI bimodal probes for in vivo imaging by enzyme-mediated fluorogenic reaction and self-assembly. J Am Chem Soc. 2019;141:10331-41.

11. Xu AL, Wang G, Li YQ, Dong H, Yang SW, He P, Ding GQ. Carbon-based quantum dots with solid-state photoluminescent: mechanism, implementation, and application. Small. 2020;16: 2004621.

12. Xia CL, Zhu SJ, Feng TL, Yang MX, Yang B. Evolution and synthesis of carbon dots: from carbon dots to carbonized polymer dots. Adv Sci. 2019;6:1901316.
13. Chen HM, Wang GD, Tang W, Todd T, Zhen ZP, Tsang C, Hekmatyar K, Cowger T, Hubbard RB, Zhang WZ, Stickney J, Shen BZ, Xie J. Gd-encapsulated carbonaceous dots with efficient renal clearance for magnetic resonance imaging. Adv Mater. 2014;26:6761-67.

14. Du FY, Zhang LR, Zhang L, Zhang MM, Gong AH, Tan YW, Miao JW, Gong YH, Sun MZ, Ju HX, Wu CY, Zou SQ. Engineered gadolinium-doped carbon dots for magnetic resonance imagingguided radiotherapy of tumors. Biomaterials. 2017;121:109-20.

15. Ren XY, Liu LH, Li Y, Dai Q, Zhang M, Jing XL. Facile preparation of gadolinium(III) chelates functionalized carbon quantum dot-based contrast agent for magnetic resonance/fluorescence multimodal imaging. J Mater Chem B. 2014;2:5541-49.

16. Xu Y, Jia XH, Yin XB, He XW, Zhang YK. Carbon quantum dot stabilized gadolinium nanoprobe prepared via a one-pot hydrothermal approach for magnetic resonance and fluorescence dualmodality bioimaging. Anal Chem. 2014;86:12122-29.

17. Wang WT, Zhang QC, Zhang M, Liu YH, Shen J, Zhou NL, Lu $\mathrm{XY}$, Zhao $\mathrm{CH}$. Multifunctional red carbon dots: a theranostic platform for magnetic resonance imaging and fluorescence imagingguided chemodynamic therapy. Analyst. 2020;145:3592-97.

18. Xu Y, Li YH, Wang Y, Cui JL, Yin XB, He XW, Zhang YK. 13Cengineered carbon quantum dots for in vivo magnetic resonance and fluorescence dual-response. Analyst. 2014;139:5134-39.

19. Bourlinos AB, Bakandritsos A, Kouloumpis A, Gournis D, Krysmann M, Giannelis EP, Polakova K, Safarova K, Hola K, Zboril R. Gd(iii)-doped carbon dots as a dual fluorescent-MRI probe. J Mater Chem. 2012;22:23327-30.

20. Ren X, Liu L, Li Y, Dai Q, Zhang M, Jing X. Facile preparation of gadolinium(iii) chelates functionalized carbon quantum dot-based contrast agent for magnetic resonance/fluorescence multimodal imaging. J Mater Chem B. 2014;2:5541-49.

21. Grobner T. Gadolinium-a specific trigger for the development of nephrogenic fibrosing dermopathy and nephrogenic systemic fibrosis? Nephrol Dial Transpl. 2006;21:1104-08.

22. Darrah TH, Prutsman-Pfeiffer JJ, Poreda RJ, Ellen Campbell M, Hauschka PV, Hannigan RE. Incorporation of excess gadolinium into human bone from medical contrast agents. Metallomics. 2009:1:479.

23. Pan D, Schmieder AH, Wickline SA, Lanza GM. Manganesebased MRI contrast agents: past, present, and future. Tetrahedron. 2011;67:8431-44.

24. Kalman FK, Nagy V, Varadi B, Garda Z, Molnar E, Trencsenyi, Kiss J, Meme S, Meme W, Toth E, Tircso G. Mn(II)-based MRI contrast agent candidate for vascular imaging. J Med Chem. 2020;63:6057-65.

25. Botár R, Molnár E, Trencsényi G, Kiss J, Kálmán FK, Tircsó G. Stable and inert Mn (II)-based and $\mathrm{pH}$ responsive contrast agents. J Am Chem Soc. 2020;142:1662-66.

26. Pujales-Paradela R, Carniato F, Esteban-Gómez D, Botta M, Platas-Iglesias C. Controlling water exchange rates in potential $\mathrm{Mn}^{2+}$-based mri agents derived from NO2A2-. Dalton Trans. 2019;48:3962-72.

27. Ji Z, Ai PH, Shao C, Wang TJ, Yan CX, Ye L, Gu W. Manganesedoped carbon dots for magnetic resonance/optical dual-modal imaging of tiny brain glioma. ACS Biomater Sci Eng. 2018;4: 2089-94.

28. Atabaev Timur SH, Piao ZL, Molkenova A. Carbon dots doped with dysprosium: a bimodal nanoprobe for mri and fluorescence imaging. J Funct Biomater. 2018;9:35.

29. Zhang R, Liu Y, Li Y, Han Q, Zhang TQ, Zeng KL, Zhao C. Preparation of polyvinylidene fluoride modified membrane by tannin and halloysite nanotubes for dyes and antibiotics removal. J Mater Sci. 2021;56:10218-30.

30. Liu T, Zhang MK, Liu WL, Zeng X, Song XL, Yang XQ, Zhang $\mathrm{XZ}$, Feng J. Metal ion/tannic acid assembly as a versatile 
photothermal platform in engineering multimodal nanotheranostics for advanced applications. ACS nano. 2018;12:3917-27.

31. Ren XY, Liu LH, Li Y, Dai Q, Zhang M, Jing XL. Facile preparation of gadolinium(III) chelates functionalized carbon quantum dot-based contrast agent for magnetic resonance/fluorescence multimodal imaging. J Mater Chem B. 2014;2:5541-49.

32. Xu WB, Mu B, Wang AQ. Facile fabrication of well-defined microtubular carbonized kapok fiber/NiO composites as electrode material for supercapacitor. Electrochim Acta. 2016;194:84-94.

33. Phuriragpitikhon J, Phinney EO, Jaroniec M. Potassium citrateassisted eco-friendly synthesis of tannin-derived nitrogen-doped micro-mesoporous carbon microspheres. J Mater Sci. 2020;55: 13716-36.

34. Liu YB, Shi QY, Zhang Y, Jing JL, Pei J. One-step facile synthesis of $\mathrm{Au} @$ copper-tannic acid coordination core-shell nanostructures served as photothermal-enhanced ROS generator for synergistic tumour therapy. New J Chem. 2020;44:19262-69.

35. Yue R, Wen X, Mao YN, Su YT, Shen Q, Song HZ, Zhang HL, Ba XW. Eco-friendly fabrication of Au nanoparticles immobilized on tannin-aminopropyltriethoxysilane-coated halloysite nanotubes for thermally tunable catalysis. J Mater Sci. 2020; 55:17094-107.

36. Shi L, Yang JH, Zeng HB, Chen YM, Yang SC, Wu C, Zeng H, Yoshihito $\mathrm{O}$, Zhang QQ. Carbon dots with high fluorescence quantum yield: the fluorescence originates from organic fluorophores. Nanoscale. 2016;8:14374-78.

37. Tian H, Zeng LM, Huang YF, Ma ZH, Meng G, Peng LX, Chen C, Cui XZ, Shi JL. In situ electrochemical $\mathrm{Mn}(\mathrm{III}) / \mathrm{Mn}(\mathrm{IV})$ generation of $\mathrm{Mn}$ (II)O electrocatalysts for high-performance oxygen reduction. Nano-Micro Letters. 2020;12:161.

38. He X, Luo Q, Zhang J, Chen P, Wang HJ, Luo K, Yu XQ. Gadolinium-doped carbon dots as nano-theranostic agent for MR/ FL diagnosis and gene delivery. Nanoscale. 2019;11:12973-82.

39. Morse SV, Boltersdorf T, Harriss BI, Chan TG, Baxan N, Jung HS, Pouliopoulos AN, Choi JJ, Long NJ. Neuron labeling with rhodamine-conjugated Gd-based MRI contrast agents delivered to the brain via focused ultrasound. Theranostics. 2020;10:2659-74.

40. ZhuW LiangS, Wang J, Yang Z, Zhang L, Yuan TM, Xu ZS, Xu $\mathrm{HB}$, Li PH. Europium-phenolic network coated BaGdF5 nanocomposites for tri-modal computed tomography/magnetic resonance/ luminescence imaging. J Mater Sci: Mater Med. 2020;28:74.

41. Liang HS, Li J, He Y, Xu W, Liu SL, Li Y, Chen YJ, Li B. Engineering multifunctional films based on metal-phenolic networks for rational $\mathrm{pH}$-responsive delivery and cell imaging. ACS Biomater-Sci Eng. 2016;2:317-25.

42. Xu WB, Mu B, Wang AQ. Three-dimensional hollow microtubular carbonized kapok fiber/cobalt-nickel binary oxide composites for high-performance electrode materials of supercapacitors. Electrochimica Acta. 2017;224:113-24.

43. Pinto SMA, Calvete MJF, Ghica ME, Soler S, Gallardo I, Pallier A, Laranjo MB, Cardoso AMS, Castro MMCA, Brett CMA, Pereira MM, Toth E, Geraldes CFGC. A biocompatible redox MRI probe based on a Mn(II)/Mn(III) porphyrin. 2019;48:3249-62.

44. del Rosal B, Benayas A. Strategies to overcome autofluorescence in nanoprobe-driven in vivo fluorescence imaging. Small Methods. 2018;2:1800075. 